\title{
Creating Data OUtputs From Multi AGENT TRAFFiC MicRo SimUlation TO ASSimilation With REAL TrafFic DATa
}

\author{
Michala Drozdova ${ }^{1}$, Petr Rapant ${ }^{1}$ and Jan Plucar $^{2}$ \\ ${ }^{1}$ Department of Geoinformatics, Faculty od Mining and Geology, \\ VSB - Technical University of Ostrava, Ostrava, Czech Republic \\ michala.drozdova@vsb.cz, petr.rapant@vsb.cz \\ ${ }^{2}$ Department of Informatics, Faculty of Electrical Engineering and Computer \\ Science, VSB - Technical University of Ostrava, Ostrava, Czech Republic \\ jan.plucarevsb.cz
}

\begin{abstract}
The intensive development of traffic engineering and technologies that are integrated into vehicles, roads and their surroundings, bring opportunities of real time transport mobility modeling. Based on such model it is then possible to establish a predictive layer that is capable of predicting short and long term traffic flow behavior. It is possible to create the real time model of traffic mobility based on generated data. However, data may have different geographical, temporal or other constraints, or failures. It is therefore appropriate to develop tools that artificially create missing data, which can then be assimilated with real data. This paper presents a mechanism describing strategies of generating artificial data using microsimulations. It describes traffic microsimulation based on our solution of multiagent framework over which a system for generating traffic data is built. The system generates data of a structure corresponding to the data acquired in the real world.
\end{abstract}

\section{KEYWORDS}

Traffic microsimulation, multiagent system, artificial traffic data, dynamic model of mobility.

\section{INTRODUCTION}

Transport telematics respectively Intelligent Transport Systems (ITS) is a branch, which concerns the use of intelligent transportation technologies and services to improve economy, safety and ecology of transport processes. Implementation of ITS in road transport increases the capacity of existing infrastructure, decreases accident rate and frequency in both normal and abnormal operating conditions. Thereby it considerably reduces the societal losses and damages due to congestions.

Effective ITS usually corresponds to extensive system of interconnected communication, information and expert technologies, which have to build a comprehensive, conceptual and organization model. Most important utility of ITS is the operation on strategic level, e.g. central traffic control system. Such system has to have sophisticated automated control, which regulates 
or proposes strategic responses, e.g. in case of unexpected events, accidents, failures. Responses to such an event can include parallel changes in traffic signal control system, usage of variable traffic signs and information boards. Traffic management must be founded on the trafficengineering analysis and appropriate concept, which is based on:

1. Reliable on-line traffic flow data (traffic flow behavior).

2. Transport models and forecasting of traffic control according to statistical analysis of historical traffic data.

Traffic data represent crucial input for traffic control systems, application provides traffic information for drivers, passengers and statistical data processing in the field of transportation planning and engineering. Thus it is essential to collect data in required quality and quantity in the Czech Republic. The lack of data may cause insufficient utilization of ITS's application. The investments in ITS may not be used effectively because of fully unutilized potential of ITS.

Road traffic modelling represents a great potential for testing new ideas and algorithms in traffic control, verifying principles of localization and prediction of congestions, or optimization of road network elements in terms of their safety and operation efficiency.

In a more global scale, application of ITS supports design of transport infrastructure and comprehensive planning. In this paper transport simulation is used as a tool for transport data generation, which is corresponding to real data and its structure in the Czech Republic.

\section{RELATED WORKS}

This section contains information about actual state of the application multiagent systems (MAS) in transportation area, traffic simulators and studies that address problem of missing traffic data.

\subsection{Application MAS in Transportation Area}

In Agent-oriented simulation systems, every element of the intelligent transportation infrastructure is modeled through an agent. This is mainly due to the fact that road traffic and transport systems are composed of many autonomous entities that show signs of intelligence. These entities such as vehicles, traffic lights, traffic signs, etc., are deployed in the network. They cover a specific part of the geographical area and interact with each other in order to meet a common goal. Paper [1] presents a comparison of a centralized hierarchical multiagent architecture with decentralized hierarchical multiagent architecture, both in the context of intelligent traffic infrastructure management in urban road network. The comparison is based on examining two systems InTRYS and TRYSA2. Da Silva with the team [2] presented a simulation tool based on the principles of microsimulation models. Robustness of this system, which works with online data and previously collected data, deals with several issues related to transport mobility. Doniec and his team [3] presented a simulation model of the behavior of vehicles at intersections. Each vehicle is represented by an agent, and coordinates its actions with other vehicles in the intersection or vehicles approaching intersection. The mechanism that controls the coordination of vehicles in the intersection is based on the perceptions of the surrounding traffic situation [4]. Ossowski and Vasirani [5] addressed the management of urban road transport using the market approach. The general idea behind their approach lies in the individual reservation of time and space at the intersection for individual vehicles. Market space, which is the space of an intersection that could be booked, is regulated by a set of specific rules for making reservations. These rules are implemented through agent interaction protocols. Paper [6] presents a bimodal model for traffic control based on MAS, which aims to verify the strategic management of duration of signals on semaphore lights, which should lead to an increase in speed of the 
intersection of public transport vehicles and all other vehicles. JADE (Java Agent DEvelopment framework) was used for the simulator implementation.

\subsection{Artificial and Missing Traffic Data}

There are different approaches to solve the problem of missing traffic data. To help improve the quality of traffic data, imputation algorithms have been developed to make estimates about missing data. Imputation techniques developed thus far can be classified into three categories: regression, nearest neighbor and deck replacement, and classifi cation. Their applications in traffic data imputation have been presented by Smith et al. in [7], Al-Deek and Chandra in [8] and Gold et al. in [9]. Researchers in [10] consider eleven algorithms for imputing missing traffic data recorded by automatic loop detectors in the Dallas, Texas region. These algorithms are compared by artificially removing a sample of original data, calibrating using the remaining data. The algorithms were then compared (eight of these existed in the pas literature and 3 were developer in this research) as to their plausibility in reconstructing the removed data. Zhong et al. [11] discussed the application of neural networks and a genetic algorithm for imputing missing traffic data from permanent count stations. Study [12] introduces a comparative analysis of various techniques for imputing missing traffic volume data in the archived data management system in Kentucky.

Experimental investigations of spatiotemporal algorithms and data structures demand for generators that produce realistic data sets. In the last few years, several generators for producing spatiotemporal data have been developed [13, 14, 15, 16]. Brinkhoff in study [17] presented two approaches for generating traffic data - the Network-based Generator and the City Simulator. Both generators allow the simulation of the motion of a huge number of moving objects. They have been integrated into more complex architectures for testing spatiotemporal queries. The Network-based Generator (written in Java 1.1.) is based on the observation that objects often move according to a network. This observation holds, e.g., for road traffic as well as for railway traffic. The generator uses a discrete time model and each moving object belongs to a class that specifies the behaviour. The network used by the generator is specified by simple text files or by spatial data stored in Oracle Spatial. The City Simulator is a scalable, three-dimensional model city that enables the creation of dynamic spatial data simulating the motion of up to 1 million moving objects. The movement of the objects is influenced by the rules of the place they are in the Network-based Generator is limited to two-dimensional data sets, the City Simulator supports three-dimensional city plans and computes $3 \mathrm{D}$ points.

\section{SimUlation}

From the global point of view there are two types of simulations: macroscopic and microscopic. Macroscopic simulations are based on standard macroscopic quantities in the context of transport sphere, e.g.: traffic flow density, intensity or speed. Particular traffic flows are recognized and evaluated in this simulation. On the other hand, microscopic simulations consider every entity of road traffic in detail and all parameters of defined entities and road infrastructure.

As aforementioned before, the aim of simulation in this paper is to generate new traffic data. This data is generated by individual road participants (vehicles). Data is detected by network of devices based on different technologies and placed in transport infrastructure. Microscopic simulation was chosen for its capability to recognize objects at the vehicles level.

An essential step in microsimulation issue is the choice of a suitable traffic model. Paper published by Wegener [18] provides an interesting summary of the problems traffic models have had and still have to face. The results of microsimulation have little informative value, because there are several ways how to solve each traffic problem. Final results then differ, depending on 
the variant that has been chosen. It is therefore necessary to repeat microsimulation for multiple variations of model evaluation and then statistically process the results.

The proposed workflow is following:

1. Creating ontology of interest domain - definition of elements participating in simulation.

2. Choosing approach under which the simulation will be designed.

3. Model designing.

4. Creating the system for road traffic simulation.

5. Traffic data sources identification, its geographical distribution and data structure.

6. Creating simulation layer of primary traffic data sources.

\section{ONTOLOGY}

The definition of ontology was formulated in many ways over time - from a philosophical point of view as the study of nature of being, according to [19] explicit specification of a conceptualization, according to [20] formal specification of a shared conceptualization or [21] hierarchically structured set of terms describing a particular substantive area. Various subontologies for different domains are created as artifact reflecting the use of an application or system.

\subsection{Ontology Description}

The ontology created for the needs of our simulations is understood as a set of knowledge about the domain of interest that will be simulated. It is formally described as [22] these six following sets: $\mathrm{O}=\left\{\mathrm{C}, \mathrm{A}^{\mathrm{C}}, \mathrm{R}, \mathrm{A}^{\mathrm{R}}, \mathrm{H}, \mathrm{F}\right\}$.

- C determines a set of concepts.

- $\mathrm{A}^{\mathrm{C}}$ represents a set of attribute collections (one collection for each concept $\mathrm{c}_{\mathrm{i}}$ ).

- $\mathrm{R}$ determines a set of relationships. Each relation in $\mathrm{R}$ is binary association between two concepts.

- $\mathrm{A}^{\mathrm{R}}$ represents a set of attribute collections (one collection for each relation $\mathrm{r}_{\mathrm{i}}$ ).

- H represents concepts hierarchy.

- F specifies constraint on the relations between concept objects or restriction and constrains on the attribute values of concepts or relationships.

\subsection{Traffic Ontology}

The main aim when constructing ontology is to capture the knowledge for specific domain. We have constructed ontology for traffic domain and it was used to design traffic microsimulation.

Base level of our traffic ontology, created to suit the needs of our next work, is composed of these four concepts: Road Network Elements, Transport Participants, Traffic Signals and Data Sources. So in this case is set $\mathrm{C}=\{$ Road Network Elements, Transport Participants, Traffic Signals, Data Sources\}. 


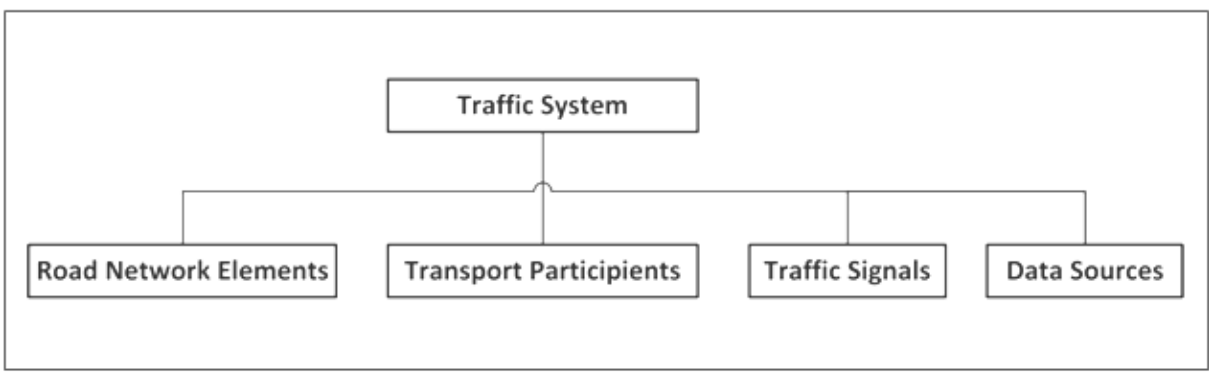

Figure 1. Ontology base level.

Each of concepts, seen in the figure Figure 1, consists of its own hierarchy of other concepts that are participating in entire ontology. See figures Figure 2, Figure 3, Figure 4 and Figure 5.

Ontology in level of detail as schematically shown in the previous figures, demonstrates only three from six elements of the formal ontology definition - concepts, relationships and hierarchy. Specification of sets $\mathrm{A}^{\mathrm{C}}, \mathrm{A}^{\mathrm{R}}$ and $\mathrm{F}$, is broad in scale and the main priority of this paper is not to explain it.

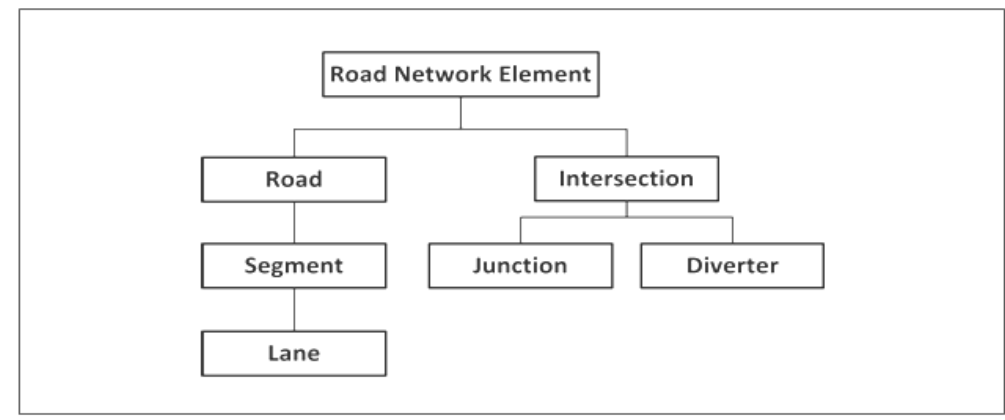

Figure 1. Road Network Element ontology.

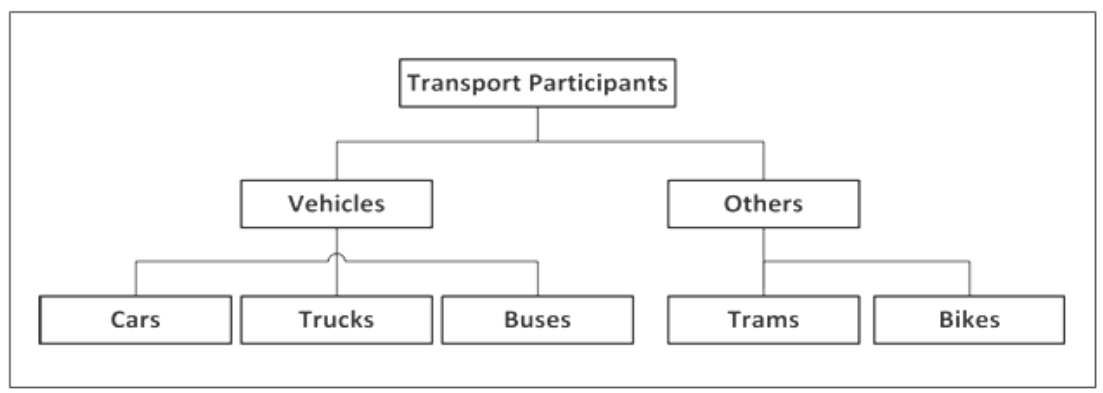

Figure 2. Transport Participants ontology. 


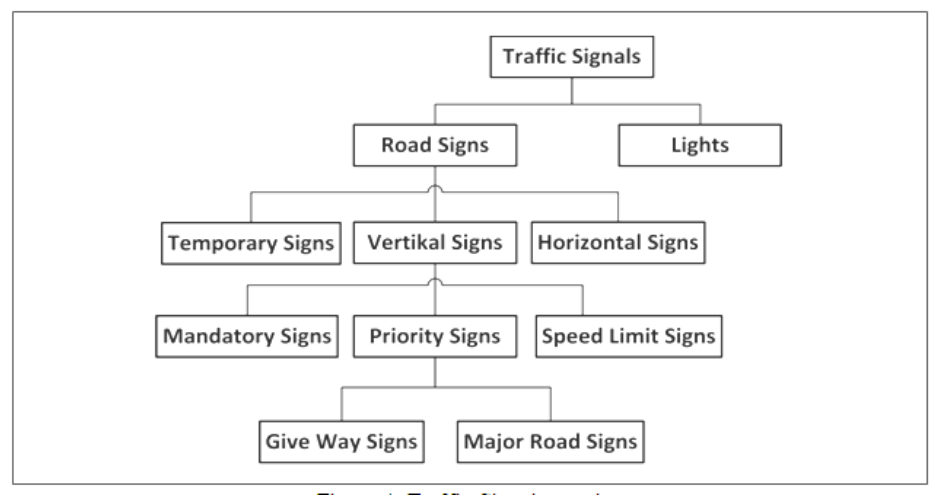

Figure 4. Traffic Signals ontology.

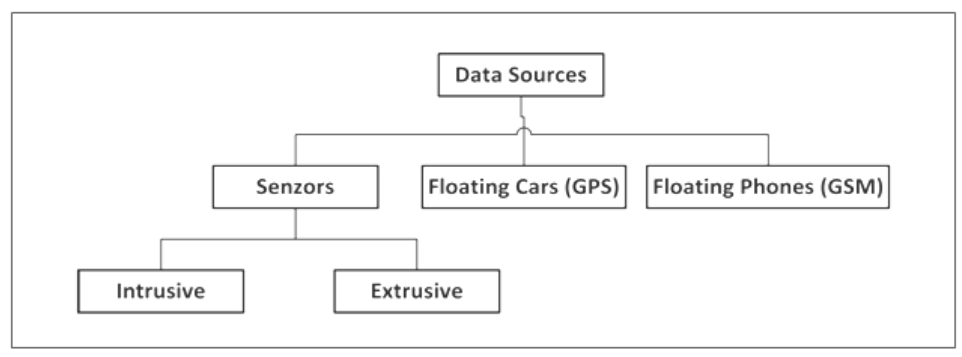

Figure 5. Data Sources ontology.

\section{Principles of Traffic Microsimulation}

In preparation for traffic microsimulation interest domain was surveyed comprehensively. Existing microsimulation solutions are based on the principles of cellular automata [23-26] neural networks [27], [28], multi-agent systems [29-33] and various combinations of these approaches [34].

Multiagent approach for microsimulation modeling was chosen according to the information gained in the research area and with regard to the intended usage. In general, transportation and transport systems are a very good example, in which it is appropriate to apply the agent based approach.

Geographical distribution, changes in characteristics describing alternating peaks and temporary inactivity are the reasons for solving computational processing of the transport sphere by agent oriented technology [35], [36].

\subsection{Agent Oriented Approach}

Road traffic and transport systems are composed of many autonomous entities that show signs of intelligence. These entities (in the context of transport are represented by vehicles, intersections, traffic lights, etc.) are deployed in the network and cover a specific part of the area. They interact with each other in order to meet a common goal.

Agent is a term for a separate entity. Agents possess the ability to perceive their environment, to communicate with environment and to make independent decisions about their following actions. 
Key characteristics of agents are autonomy and integration in environment [37] which they are able to act autonomously [38].

Multiagent system is a set of agents that represent active components of the modeled system and are able to interact in order to achieve the general objectives [39].

Transportation problem could be naturally decomposed into sub-units (agents) that interact with each other in order to achieve the overall goal. To be able to use multiagent modeling approach, it is necessary for target domain to meet the following three conditions [40]:

- Target domain is geographically distributed.

- The environment, in which subsystems exist, is dynamically changing.

- Flexible mutual interaction is required between subsystems.

Road traffic and transport systems are composed of many autonomous entities that show signs of intelligence. These entities are deployed in the network and cover a specific part of the area. They interact with each other in order to meet a common goal. In Agent oriented simulation transport systems is every single element of intelligent transportation infrastructure modeled through an agent. Multiagent approach for microsimulation modeling was chosen according to the information gained in the research area and with regard to the intended usage. In general, transportation and transport systems are a very good example, in which it is appropriate to apply the agent based approach.

\section{Multiagent Framework Architecture}

There are several agent oriented architectures, which are generally used in multiagent modeling. Each of these architectures is suitable for different task. In our case, the most suitable architecture is the hierarchical one, in which the hierarchy of agent's roles is the most essential element. Central management element is also part of this architecture, but it does not perform all tasks itself. It may delegate part of the communication and management tasks to the control elements in lower levels of the hierarchy. Such architecture can be represented in the form of a tree, see Figure 6. Leaves of the tree represent discrete and finite agents. The advantage of this architecture is scalability and robustness. Adding additional control elements supports load balancing management. New element can be simply added as a child of its parent agent and there is no need to change the implementation of system.

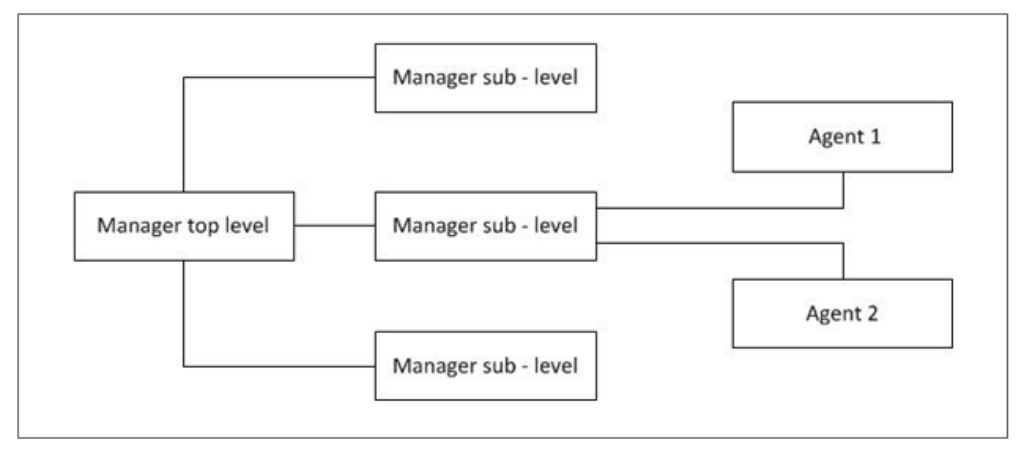

Figure 6. Hierarchical architecture.

In terms of development, deployment and extension of practical applications on multi-agent principles, it is important to support standards in unified architecture design of multiagent applications. The solution is implemented and performed according to FIPA standard (Foundation 
for Intelligent Physical Agents). FIPA is an international respected standard, which promotes interoperability and reusability of multiagent systems and defines the key elements of architecture that must be implemented.

In this study own general architecture of multiagent framework was designed. This framework was implemented in C\# language with usage of object-oriented methodology, follows principles of hierarchical architecture and requirements of multiagent system, which are declared in the FIPA standard. There were several design patterns such as composite, creator, factory, respectively abstract factory or singleton used.

\subsection{Core of Designed Architecture}

Class diagram, see Figure 7, represents specific architecture of a multiagent system. This diagram reflects only the core of the system. Some classes are not displayed due to the lack of space. The core design elements are classes Manager, Agent and Structure.

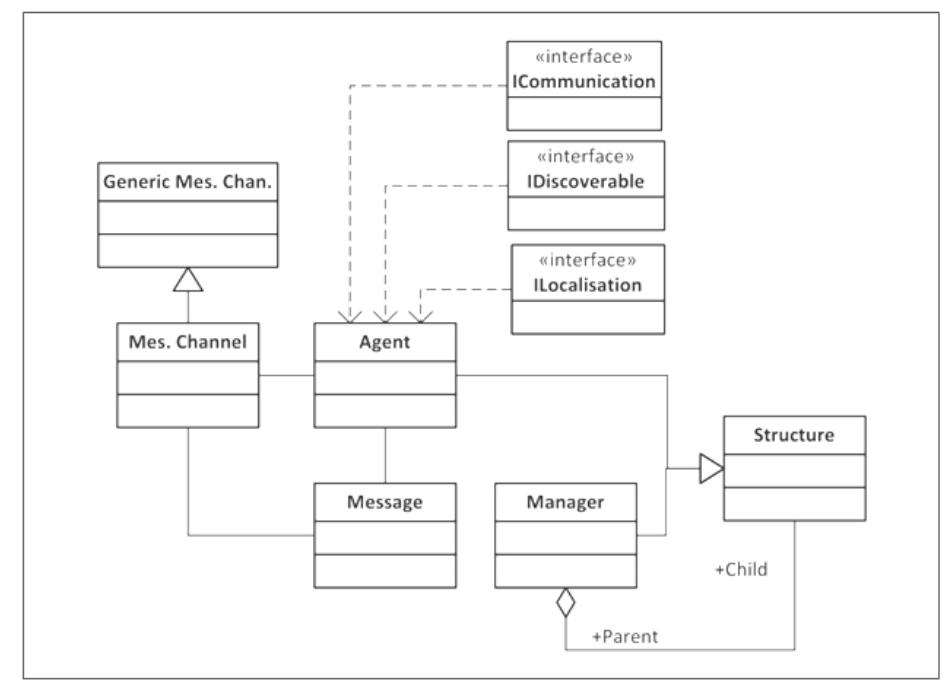

Figure 7. Core of the architecture.

Manager, Agent, Structure: these classes represent hierarchical organization of agents, specifically utilizable for elementary geofraphical areas in simulated region.

Generic Message Channel a Message Channel: communication channel for all messages sent. ICommunication: declares definitions of methods, which are used for establishing of connection to message channel and creating and sending messages (stated in FIFA).

IDiscoverable: declares definitions of methods, which are responsible for searching, adding, removing agents from agent storage (stated in FIFA).

ILocalisation: declares definitions of methods, which are responsible for searching services in service storage (stated in FIFA).

\subsection{Messaging in Framework}

Design pattern creator was applicated for effective realization of message creation mechanism. Communication between agents is implemented in three different ways: 
1. socket communication - protocol TCP,

2. socket communication - protocol UDP,

3. inter-object message sending,

whereby the possibilities of using the framework for MAS implementation with agents distributed in network or undistributed implementation is regarded - realization on one computation station.

Every agent starts and runs both TCP client and TCP server in order to be able to receive and send messages. Both TCP server and TCP client of each agent are launched in their own thread so that running them does not oppress the main computational thread of the agent.

Message object is a communication element that uses XML format for the socket communication. The Message consists of Header and Content. The Header contains information about a sender and a receiver (IP address, port, etc.). The Content contains specific information which is the subject of the communication. A message can be encrypted before it is sent and decrypted when received. The MD5 algorithm is used for this encryption and it may as well be replaced by any other encryption algorithm. Finally, the message is converted into a binary form and sent.

\section{Agent Model}

An agent model described below was designed for traffic microsimulation. This model specifies the interactions and relations among agents within the whole multiagent system and provides an external view of a specific multiagent system as a whole. Its content is a definition of a hierarchy of agent classes and their interactions - this is schematically described at Figure 8.

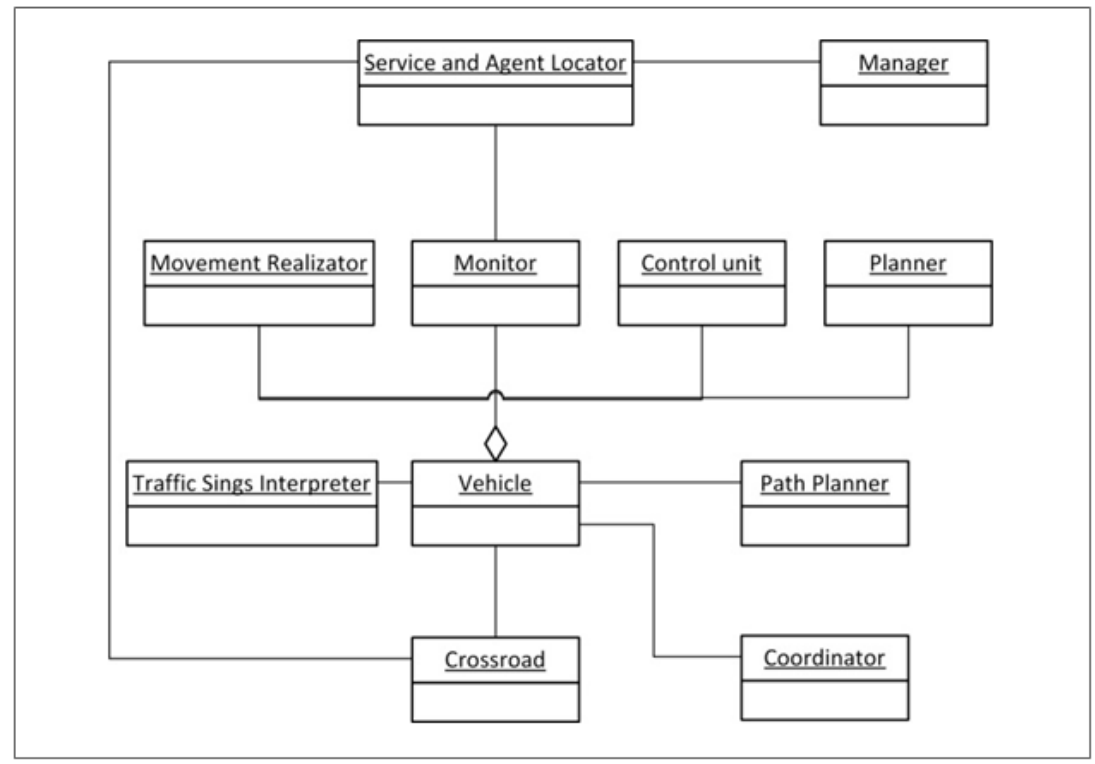

Figure 8. Agent model.

Following types of agents were defined within this agent model:

Vehicle - agent representing means of transport, which are moving within the road network. Every agent vehicle is composed of other support agents that solve following fragmental tasks: 
- Monitoring of the surroundings and data gathering about other vehicles (agents) - Agent Monitor.

- Data acquisition about traffic communication.

- Performing movement, i.e. recalculation of spatial coordinates of its location - Agent Movement.

- Making decisions based on submitted data. Such decisions may be, for example the manoeuvre of overtaking slow cars, transferring to another lane etc. - Agent Decision maker.

Crossroad (Intersection) - receives requests of particular vehicle agents for riding through the crossroad in a given direction. It deals with evaluation of a traffic situation and calculation of the order in which the cars will ride through the crossroad. The calculation differs according to each type. There are crossroads with and without traffic lights and these belong among the basic types. The Crossroad contacts the Vehicle agent as soon as it can ride through the crossroad.

Traffic signs interpreter - the Vehicle agents will call the interpreter with a request to explain the meaning of traffic signs. Information about traffic signs is contained within the communication's geographic data. For example, the interpreter receives a request to explain a traffic sign, which sets the speed limit to $50 \mathrm{~km} / \mathrm{h}$. The answer for the car agent could be: SET MAX SPEED 50.

Path planner - the vehicle agent is responsible for reading the road segment, where the Vehicle agent is located. However, it is redundant to map and record the whole journey from the start till the destination. The agent will keep a list of the closest segments. This list is regularly updated by the Path planner agent, which is called by the agent every time it passes one of the road segments.

Service and Agent locator - this agent takes care of connecting to a service and agent database. The other agents use it to query for a specific agent or service.

Director (manager) - a managing agent for a particular area. Its main task is to manage traffic fluency. For example, it controls if a crossroad agent is up and running.

Coordinator - this agent takes care of the simulation process by generating car agents and removing those, who have already participated in the simulation. While creating a vehicle agent a path, which the agent will follow, must be designed.

\section{Simulation EnVIRONMENT}

The insights of the real world traffic situation are traffic simulations that are realized by the proposed multiagent framework. This intention is the basis of the idea that the simulation environment must necessarily be spatial and agents must have the ability of situational localization.

Multiagent Situated System (MASS) introduces situated agents that are sensitive to their position and spatial properties, which may restrain or privilege their interactions. Localization of agents reflects their position and spatial relationships among particular [41].

Geographic data was used to realize the spatial simulation environment. Geographical information systems normally operate in two-dimensional space, which can be represented as: 
- metric,

- topological.

Metric two-dimensional space is used in the cases, when the knowledge of precise absolute position expressed by coordinates, shape or size is needed. On the other hand, topological space is mainly used when the expression (or analysis) of mutual spatial relations of objects are needed [42].

\subsection{Object Representation of Environment}

Agents connected with the area of transport infrastructure need to perform various computations over geographic data. While preparing environment for agents, it is necessary to solve the two following tasks as shown in Figure 9.

1. Prepare geographic data representing selected area of the real world over which the simulation will be carried out

2. Geographic data is transferred into the simulation environment in the form of object.

Environment in which the multiagent system will be set must represent the road network over which microsimulation models are implemented. For this purpose analysis of the structure and content of data was performed on the sample data provided by CEDA (Central European Data Agency). Attributes that were found unnecessary for the creation of microsimulation were excluded, which was based on the analysis. Data was purified by its own data pump and its structure was modified to allow the possibility to design a database over this data according to the third normal form. An object-relational mapping was performed over this database which resulted in the object representation of an environment for the agents - the object representation of the road network.
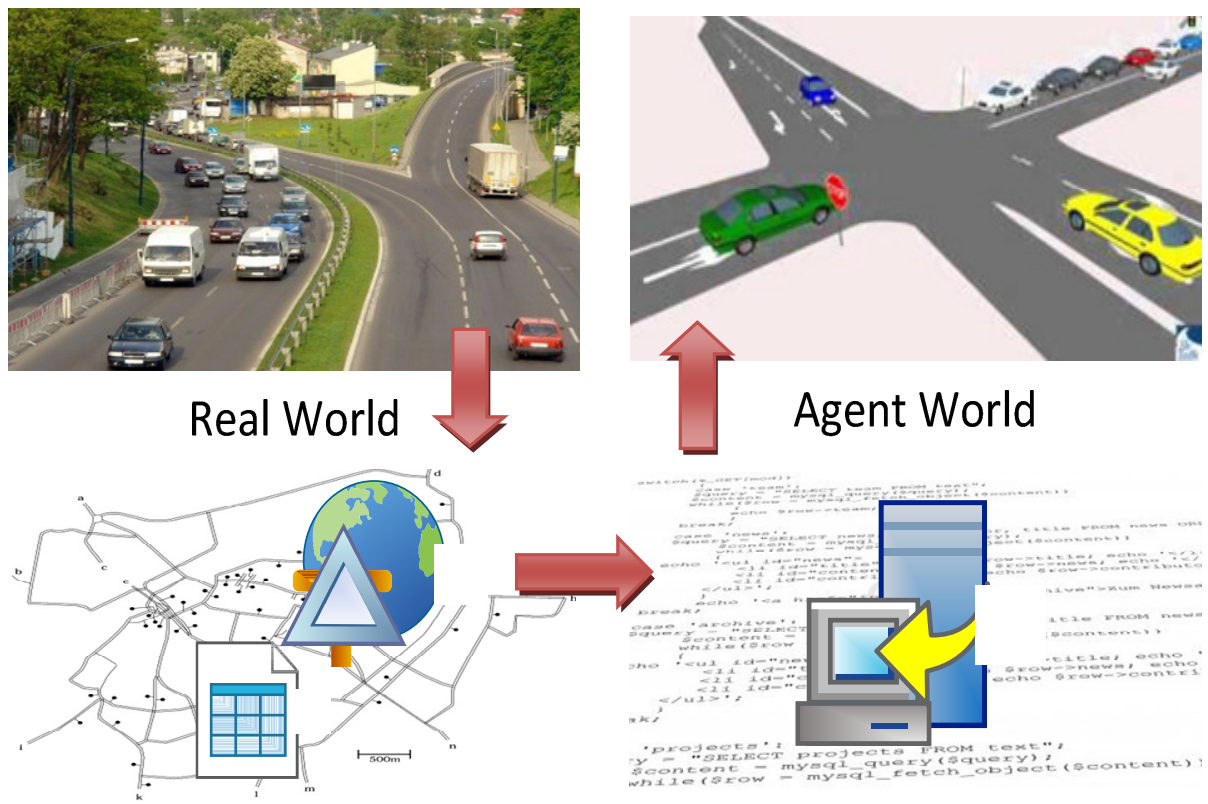

GIS Data

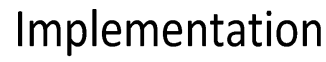

Figure 9. Simulation environment process. 


\section{Simulation SySTEM EXTENSION}

Another step in the solution is to create a layer implementing a mechanism, which generates data over the traffic background simulation system. This data documents the traffic.

Workflow process:

1. Identification of the traffic data primary sources captured in the traffic infrastructure.

2. Specification of the geographic detector placement is a simulated area.

3. Generated data format specification.

4. Suggestion of the appropriate data structure for saving the generated data.

\subsection{Primary Data Source Identification}

There are several data collection technologies, which describe the phenomena connected with mobility. Based on these technologies study, four following types of data were identified. This data is registered in the traffic infrastructure.

\subsubsection{Profile Detector Data}

Profile detectors used in the Czech Republic can be divided to intrusive and non-intrusive. Intrusive sensors are located on the surface of the roadway or inside of it (pneumatic sensors, piezoelectric, magnetic and induction loops. Non - intrusive sensors are located over or next to a communication. They monitor the traffic without an intervention in the roadway (video-detection, microwave radars, acoustic sensors and infra-light sensors).

\subsubsection{Toll System Data}

Only vehicles over 3,5 $\mathrm{t}$ are electronically charged in the Czech Republic. Vehicles under 3,5 are assumed to be charged by 2016, which would mean that the toll system would include every vehicle on the charged communication. Thus, the traffic data conducted by the toll gates system will document the movement of all traffic flow vehicles. Blind spots may occur as the measurement is still mainly profile.

\subsubsection{Floating Car Data - FCD}

So called floating vehicles are an appropriate supplement of the existing traffic data profile sources, which provide data on GPS system basis and Galileo system in the future.

\subsubsection{Floating Phone Data}

Floating Phone Data is GSM mobile network signalizing data that monitor the movement of mobile phones in the mobile network.

All this data is complementary and combining it sufficient quantities of reliable traffic data for the road network can be obtained. 


\subsection{Artificial Data}

Data format of artificially generated data will be inspired by real data format, which is captured while monitoring transport mobility. This is particularly important in order to process artificially generated data in the same way as the real data.

Data describing the phenomena associated with the transport mobility have certainly spatiotemporal nature and working with them is not trivial. The design process of optimal data structure into which the generated data is stored, should not therefore be underestimated, not only because of their spatio-temporal character, but also because of the volume of data that will directly reflect the size of the simulated area generated.

\subsection{Generating Data Mechanism}

Implementation of the extension above the basic traffic simulator tool is also designed using multiagent approach. Layer for generating the data described in this chapter is separate multiagent system, which shares the same simulation environment with the original simulator.

The individual data sources are performed by agents that monitor their surroundings, which is determined by the size and shape of their sensoric field. If there is agent of certain type registered in the surrounding of monitoring agent, data record is generated.

\section{RESULTS}

Within a unique project called IT4Innovations, which is a part of international supercomputer expert workplace site PRACE (Partnership for Advanced Computing in Europe), a supercomputer center is created in the Czech Republic in the campus of Vysoká škola báňská Technical University of Ostrava (VŠB-TUO).

In order to increase competitiveness of the Czech Republic in the ITS field a competence center RODOS was created under the auspices of VSB - TUO or IT4I. Apart from the academic community, experts in the field of traffic telematics participated on its development. The Dynamic mobility model is one of the planned outputs of this center. The purpose of this model is to perform a complex analysis of model that reflects the movement of people and goods based on actual traffic data. Its outputs will support planning and operational processes within the traffic system of the Czech Republic.

The use of data collection technologies in the Czech Republic is minor. A current situation of the area which is not covered by the sensors is not possible to be monitored. In such situation, it is appropriate to use simulations, which enable us to estimate artificial data with a sufficient probability. Artificially generated data is adequate to the data actually registered in a traffic infrastructure, which is the output of the microsimulation model described above. This artificial data will complement the real data and complete and refine the DMM. See Figure 10. 


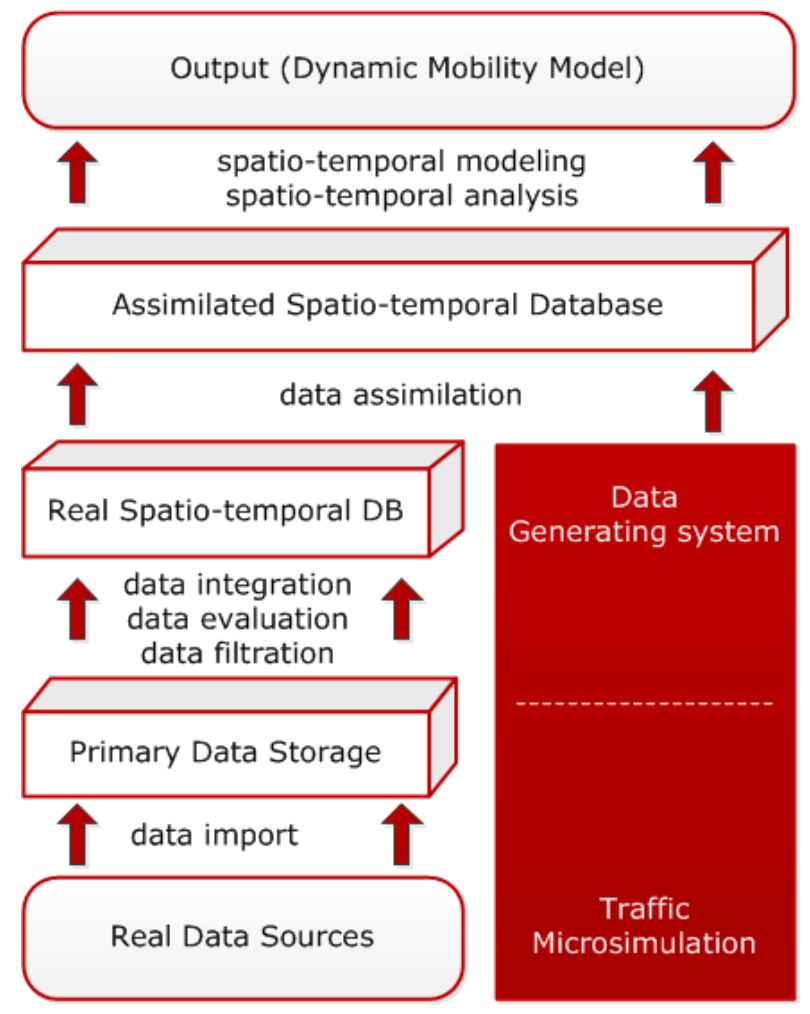

Figure 10. Scheme of simulator's using.

\section{CONCLUSIONS}

The prime function of the simulator described in this paper is to generate artificial traffic data describing phenomena associated with mobility.

The simulator is designed and implemented using multiagent approach and is composed of two subsystems. The first system simulates traffic on the selected area. It organizes the movement of vehicles in accordance with the rules of the road traffic in the simulation environment represented by the road network in the territory.

The second system implements network devices in the same simulation environment. These devices are designed to capture traffic data generated by the first system. Based on this data the system generates data records corresponding to the records of actual traffic. This data will then be assimilated with real data and will have its share of dynamic mobility model that will be the output of the RODOS centre that was mentioned in the previous chapter.

There have been many bigger or smaller traffic simulator tools developed. Some of them are still under development and the resulting tools both commercial and open source are becoming more robust. Reason behind the design and development of new tool for traffic simulations and multiagent framework is mainly the openness of the whole system. Developing a custom tool provides the ability to extend or modify the tool completely including performance or throughput optimization. Moreover we are able to alter application logic when necessary. We will benefit from the full control over the simulation tool while working on the IT4I project that brings a significant computing potential. 
Tasks to be addressed in the subsequent period includes creation of validation schemas and validation of simulation system, optimizing storage of generated data with regard to its nature and spatio-temporal characteristic. Also continuous refining of simulated traffic behaviour.

\section{ACKNOWLEDGEMENTS}

This work was supported by the European Regional Development Fund in the IT4Innovations Centre of Excellence project (CZ.1.05/1.1.00/02.0070) and by Student Grant Competition of VSB-Technical University of Ostrava, project SP2013/141.

\section{REFERENCES}

[1] J. Z. Hernandez, S. Ossowski, A. Garcia-Serrano, "Multiagent architectures for intelligent traffic management systems". Transp. Res. Part C: Emerging Technol. October-December 2002, Vol. 10, 5/6, pp. 473-506.

[2] B. C. Da Silva, et al., "ITSUMO: An intelligent transportation system for urban mobility," Proc. Innovative Internet Community, Syst. Vol. 3473, pp. 224-235, 2006.

[3] A. Doniec, et al., "A behavioral multi-agent model for road traffic simulation," Eng. Appl. Artif. Intell. December 2008, Vol. 21, 8, pp. 1443-1454.

[4] R. Mandiau, et al., "Behaviour based on decision matrices for a coordination between agents in a urban traffic simulation,” Appl. Intell. April 2008, Vol. 28, 2, pp. 121-138.

[5] M. Vasirani, S. Ossowski, "A market-inspired approach to reservation-based urban road traffic management,” Proc. 8th Int. Conf. AAMAS, Budapest, Hungaria : s.n., 2009, pp. 617-624.

[6] N. Bhouri, S. Haciane, F. Balbo, "A multi-agent system to regulate urban traffic: Private vehicles and public transport," Intelligent Transportation Systems (ITSC),2006, pp. 1575-1581.

[7] B. Smith, W. Scherer, and J. Conklin. "Exploring Imputation Techniques for Missing Data in Transportation Management Systems.” Transportation Research Record: Journal of Transportation Research Board 1836, (2003): 132-142.

[8] H. Al-Deek, C. Chandra. "New Algorithms for Filtering and Imputation of Real-Time and Archived Dual-Loop Detector Data in I-4 Data Warehouse." Transportation Research Record: Journal of the Transportation Research Board 1867, (2004): 116-126.

[9] D. Gold, S. Turner, B. Gajewski and C. Spiegelman. "Imputing Missing Values in ITS Data Archives for Intervals under 5 Minutes," 80th Annual Meeting of Transportation Research Board, January 7-11, 2001, Washington, D.C.

[10] S. D. Boyles, S. T. Waller, "A Comparison of Interpolation Methods for Missing Traffic Detector Data," Transportation Research Board Annual Meeting, 2011.

[11] M. Zhong, S.Sharma, and P. Lingras. "Genetically Designed Models for Accurate Imputation of Missing Traffi c Counts." Transportation Research Record: Journal of the Transportation Research Board 1879, (2004): 71-79.

[12] M. Chen, J. Xia, R. Liu, "Developing a Strategy for Imputing Missing Traffic Volume Data,“ Journal of the Transportation Research Forum, Vol. 45, No. 3 (2006), pp. 57-75.

[13] Y. Theodoridis, J.R.O. Silva, and M.A. Nascimento. "On the Generation of Spatiotemporal atasets," In: Proc. 6th International Symposium on Large Spatial Databases, Hong Kong, China, pp. 147-164, 1999.

[14] D. Pfoser and Y. Theodoridis. "Generating Semantics-Based Trajectories of Moving Objects," In: Proc. International Workshop on Emerging Technologies for Geo-Based Applications, Ascona, witzerland, pp. 59-76, 2000.

[15] J.-M. Saglio and J. Moreira. Oporto. "A Realistic Scenario Generator for Moving Objects.,"GeoInformatica 5(1):71-93, March 2001.

[16] T. Tzouramanis, M. Vassilakopoulos, and Y. Manolopoulos. "On the Generation of Time-Evoling Regional Data," GeoInformatica, 6(3):207-231, September 2002.

[17] T. Brinkhoff, "Generating Traffic Data," Bulletin of the Technical Committee on Data Engineerin,IEEE Computer Society, Vol. 26, No. 2, 2003, 19-25.

[18] M. Wegener, "From Macro to Micro - How Much Micro is too Much?," International Seminar on Transport Knowledge and Planning Practice, Amsterdam : University of Amsterdam, 2009. 
[19] T. Gruber, "A Translation Approach to Portable Ontology Specifications," in: Knowledge Acquisition, 5(2):199-220, 1993.

[20] W. Borst, Construction of Engineering Ontologies for Knowledge Sharing and Reuse: Ph.D. Dissertation, University of Twente.

[21] B. Swartout, P. Ramesh , K. Knight , T. Russ, "Toward Distributed Use of Large-Scale Ontologies," in Farquhar A, Gruninger M, Gómez-Pérez A, Uschold M, van der Vet P (eds) AAAI'97 Spring Symposium on Ontological Engineering. Stanford University, California, 1997, pp 138-148.

[22] Tao Feng, Hong Liang, Jianping Wu, "Ontology Driven Data Sharing Between Microscopic Traffic Simulation and GIS”, IEEE International Conference on Computer Simulation and GIS, Vol. 3, 2010, pp 84-89.

[23] I. Spyropoulou, „Modelling a signal controlled traffic stream using cellular automata,“ in Transportation Research Part C: Emerging Technologies, pp. 175-190, 2007.

[24] Y.-S. Han a S.-K. Ko, „Analysis of a cellular automaton model for car traffic with a junction,“ Theoretical Computer Science, 2012.

[25] C. Mallikarjuna and K. Rao, "Identification of a suitable cellular automata model for mixed traffic," Journal of East Asian Society for Transportation Studies 7, pp. 2454-2468, 2007.

[26] R. Wang and H. J. Ruskit, "Modelling Traffic Flow at a Multilane Intersection," Computational Science and Its Applications, 2003.

[27] C. Ledoux, "An Urban Traffic Model Integrating Neural Networks," Transportation Research, vol. 5, no. 5, pp. 287-300, October 1997.

[28] D. Srinivasan, M. C. Choy and R. L. Cheu, "Neural Networks for Real-Time Traffic Signal Control," IEEE Transaction on Intelligent Trasportation Systems, vol. 7, no. 3, pp. 261-272, September 2003.

[29] V.R. Tomas and L. A. Garcia, "Agent-based management of non urban road meteorological incidents," Proc. Multi-Agent Syst. Appl. IV, pp. 213-222, 2005.

[30] A Donec, R. Mandiau, S. Piechowiak and S. Espie, "A behavioral multi-agent model for road traffic simulation," Eng. Appl. Artif. Intell., vol. 21, no. 8, pp. 1443-1454, December 2008.

[31] N. Bhouri, S. Haciane and F. Balbo, "A multi-agent system to regulate urban traffic: Private vehicles and public transport," in Intelligent Transportation Systems (ITSC), 2010.

[32] A Cicortas and N. Somosi, "Multi-Agent System Model for Urban Traffic Simuation," in 2nd Romanian-Hungarian Joint Symposium on Applied Computational Intelligence, Timisoara, 2005.

[33] D. Meignan, O. Simonin and A. Koukam, "Simulation and evaluation of urban bus-networks using a multiagent approach," Simul. Model. Pract. Theory, vol. 15, no. 6, pp. 659-671, July 2007.

[34] S. Panwai and H. Dia, "Neural agent car following models," IEEE Transaction on Intelligent Transportations Systems, vol. 8, no. 1, pp. 60-70, March 2007.

[35] F. Y . Wang, "Agent-based control for networked traffic management", IEEE Intelligent Systems. 2005, Vol. 20, 5, pp. 92-96.

[36] F. Y. Wang,"Toward a revolution in transportation operations: AI for complex systems," IEEE Intelligent Systems, vol. 23, no. 6, pp. 8-13, November/December 2008.

[37] A. U.Frank, S. Bittner, M. Raubal, Spatial and Cognitive Simulation with Multi-agent Systems. COSIT, Vienna, 2001.

[38] M. Wooldridge, "Intelligent Agents," in: G. Weiss (ed.), Multiagent systems - A modern Approach to Distributed Artificial Intelligence, MIT Press., Cambridge, MA, 1999. Str. 27-77.

[39] M. Wooldridge, "An Introduction to MultiAgent Systems", John Wiley \& Sons Ltd., Chichester, 2002.

[40] J. L. Adler and V. J. Blue, "A cooperative multi-agent transportation management and route guidance system," Transportation Research Part C: Emerging Technologies. 2002, Vol. 10, 5/6, pp. 433-454.

[41] D. Weyns, T. Holvoet.: Model for Simultaneous Actions in Situated Multi-Agent Systems. In Schillo, M. et al. (eds.): MATES 2003. Springer.Verlag Berlin Heidelberg, 2003. Str. 105-118.

[42] P. Rapant, "Prostor V multiagentových systémech modelujících prostorové procesy," Acta Montanistica Slovaca, Košice, Ročník 12(2007), číslo 2, 84-97. 


\section{Authors}

Ing. Michala DROZDOVA was born in 1987. In 2011, she finished master studies at VSB-Technical University of Ostrava, Faculty of Electrical Engineering and Computer Science, Dept. of Computer Science and Engineering. She is currently Ph.D. student. Her main interests are multi-agents systems, traffic modeling and disaster management.

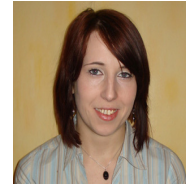

doc. Ing. Petr RAPANT, CSc. was born in 1960. He is associated professor of geoinformatics at VSB-Technical University of Ostrava, Czech Republic. His research area is geoinformatics, spatiotemporal data struc-tures and ontology, and transport modeling.

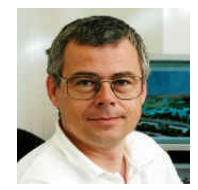

Ing. Jan Plucar was born in 1987 in Karvina. In 2011, he finished master studies at VSB-Technical University of Ostrava. He is currently Ph.D. student. His main interests are bio-inspired computing, software engineering and software process methodologies. 